\title{
Assessing the impact of a novel house design on the incidence of malaria in children in rural Africa: study protocol for a household randomized controlled trial.
}

\section{Salum Mshamu}

CSK: Centralsjukhuset Kristianstad

\section{Arnold Mmbando}

Ifakara Health Research and Development Centre: Ifakara Health Institute

Judith Meta

self employed

\section{John Bradley}

LSHTM: London School of Hygiene \& Tropical Medicine

Thomas Chavalier Bøjstrup5

KADK: Det Kongelige Akademi - Arkitektur Design Konservering

Nicholas PJ Day

MORU: Mahidol Oxford Tropical Medicine Research Unit

\section{Mavuto Mukaka}

MORU: Mahidol Oxford Tropical Medicine Research Unit

\section{Fredros Okumu}

Ifakara Health Research and Development Centre: Ifakara Health Institute

\section{Ally Olotu}

Ifakara Health Research and Development Centre: Ifakara Health Institute

Christopher Pell

Amsterdam UMC - Locatie AMC: Amsterdam UMC Locatie AMC

Jacqueline Deen

University of the Philippines Manila

Jakob Knudsen

KADK: Det Kongelige Akademi - Arkitektur Design Konservering

\section{Steven W Lindsay}

University of Durham: Durham University

Lorenz von Seidlein ( $\sim$ lorenz@tropmedres.ac)

MORU Tropical Health Network: Mahidol Oxford Tropical Medicine Research Unit https://orcid.org/0000-0002-0282-6469 


\section{Research Article}

Keywords: Housing, house screening, malaria, respiratory infections, diarrhoea, Tanzania, Africa

Posted Date: February 10th, 2022

DOI: https://doi.org/10.21203/rs.3.rs-1262951/v1

License: (c) (i) This work is licensed under a Creative Commons Attribution 4.0 International License. Read Full License 


\section{Abstract}

\section{Background:}

Traditional rural housing in hot, humid regions of sub-Saharan Africa usually consists of single-level, poorly ventilated dwellings. Houses are mostly poorly screened against malaria mosquitoes and limited airflow discourages the use of bednets resulting in high indoor transmission. This study aims to determine whether living in a novel design house with elevated bedrooms and permeable screened walls reduces malaria among children in rural Tanzania.

\section{Methods/study design:}

This is a household-randomized, controlled study in 60 villages in Mtwara, Tanzania. A total of 550 households are randomly selected, 110 of which are allocated a novel design house and 440 households continue to reside in traditional houses. A dynamic cohort of about 1,650 children under 13 years will be enrolled and followed for 3 years, approximately 330 living in novel design house s and 1,320 in traditional rural houses. The primary endpoint is the incidence of malaria, secondary endpoints are incidences of acute respiratory tract infections and diarrhoea diseases detected by passive and active surveillance. Exposure to malaria mosquitoes will be assessed using light traps in all study houses. Structural, economic, and social science studies will assess the durability, cost-effectiveness and acceptability of the new houses compared with traditional housing. Environmental data will be collected indoors and outdoors in study homes to assess differences between house typologies.

\section{Discussion:}

This is the first randomized-controlled trial to assess protective efficacy of a new house design targeting malaria in sub-Saharan Africa. The findings of this study could influence the future construction of homes in hot and humid zones of Africa.

\section{Trial registration: NCT04529434}

\section{Background}

The population of Africa, currently around 1.3 billion, is projected to double by 2050 and reach more than 4 billion by $2100^{1}$. This population growth will create demand for healthier, more comfortable, and more affordable homes. Sustainable Development Goal 11 proposes adequate, safe, and affordable housing for everyone by $2030^{2}$. To achieve this goal, it will be important to know what constitutes a healthy living space.

Traditional rural house construction in hot and humid regions of Sub-Saharan Africa often consists of wattle and daub with a thatched roof resulting in single-level, poorly ventilated spaces which can place children at risk of a range of diseases such as malaria, respiratory tract infections and diarrhoea ${ }^{3}$. Walls constructed of heavy materials with small or absent windows result in an uncomfortable indoor climate 
that is hot during the night and even hotter during the day. These conditions hamper the use of insecticide-treated nets (ITNs), which further attenuates airflow by a mean of $60 \%{ }^{4}{ }^{5}$. Compared to elevated bedrooms, single story buildings harbour higher mosquito densities ${ }^{6}$. Recently it has been demonstrated that house entry by malaria mosquitoes can be reduced substantially by elevating the house off the ground ${ }^{7}$ and by increasing ventilation to reduce indoor levels of carbon dioxide to outdoor levels ${ }^{8}$, making it difficult for mosquitoes to locate a bloodmeal and transmit malaria. Indoor air pollution mostly due to smoke from open fires but also due to dust and allergens from earth floors, muddy walls and thatched roofs increases the risk of respiratory tract infections $s^{9,10}$. Poor water supply and sanitation predispose to diarrhoeal diseases ${ }^{11-13}$.

The novel house design evaluated in this study addresses these environmental health risks. The underlying concept for the design is derived from traditional rural Asian house designs, which are elevated and constructed of light, air-permeable materials that facilitate airflow to minimize contact with vectors $^{3}$. A pilot study of six prototype houses carried out in Magoda, Tanzania in 2015, found that mosquito entry was reduced by $95 \%$ in double-story buildings and by $70 \%$ in screened single-story buildings compared with unmodified reference houses ${ }^{14}$. Both single and double story houses were $2.3^{\circ} \mathrm{C}$ (95\% Cl $2.2-2.4)$ cooler compared with traditional houses. The observed reduction in mosquito densities and temperature suggest that double story screened houses could protect best against malaria by providing a barrier to mosquito entry and encouraging bednet use in the cooler indoor environment. By promoting airflow and installing chimneys that reduces indoor smoke inhalation, the risk for respiratory tract infections could be reduced. Rainwater collection systems and hygienic, fly-proof latrines could reduce the risk of diarrheal disease transmission.

\section{Study Objectives}

Primary objective: The primary clinical objective of the study is to assess over a follow-up period of 3 years whether living in a novel design house will reduce the incidence of malaria in children compared to children living in traditional sub-Saharan African homes.

\section{Secondary objectives:}

- To determine whether living in novel design houses reduces incidence of respiratory tract infections in children.

- To determine whether living in novel design houses reduces incidence of diarrhoea diseases in children.

- To determine whether living in novel design houses reduces Plasmodium falciparum parasite rates in children.

- To assess whether living in novel design houses results in improved childhood growth by reducing the incidence of illness. 
- To determine whether living in novel design houses decreases over-all childhood hospitalisation rates.

- To identify and compare the predominant pathogens causing respiratory tract and diarrhoeal diseases between study arms.

- To compare the numbers of malaria mosquitoes and domestic flies entering novel design and traditional houses.

- To assess the use and acceptability of the novel design houses.

- To compare the frequency of ITNs use is both study arms.

- To assess the potential cost-effectiveness of the novel design house.

- To compare indoor temperature, relative humidity, carbon dioxide and atmospheric particulate matter with a diameter of less than 2.5 micrometers $\left(\mathrm{PM}_{2.5}\right)$ concentrations in both study arms.

- To compare the relative aerosol dispersion rates between study arms.

- To assess the durability of the various components of the novel design house.

\section{Methods And Study Design}

\section{Study area}

The study site is in the Mtwara region, on the south-east coast of Tanzania, which shares its southern border with Mozambique. It is an area of forest and scrubland with a very high population adjusted $P$. falciparum parasite rate standardised to the age group 2-10 years (PAPfPR 2-10>30\%) ${ }^{15}$. The climate consists of two rainy seasons: the long rains between February and April and the short rains from late October to December. The lush, green vegetation in the area is replaced by a dry, barren landscape between the rains. Mtwara has an area of $16,710 \mathrm{~km}^{2}$ and is comprised of five Districts and nine councils with a population of $1,424,083$ people in $2018^{16}$. Mtwara region is a leading cashew producer in Tanzania and more than $90 \%$ of its residents are engaged in cashew production, with a small number of fishers ${ }^{17}$. The study will be conducted in 60 villages with households that meet the inclusion criteria of the study (see below) randomly selected from Mtwara's 110 villages. The study population has no prior experience with health research.

\section{Study design}

This is an open-label, household-randomized, controlled trial using a generalized, randomised, complete, block design, with the village as a block. The study arms consist of 110 novel-design houses and 440 traditional African homes.

\section{Intervention}

The intervention consists of a novel house design. Its structure is informed by pilot studies in Magoda, Tanzania ${ }^{14}$. The critical components are an elevation of the sleeping area by constructing double-storey 
buildings and the use of shade-net as cladding to optimize airflow while minimizing the entry of insects (Figure 1). To assure timeliness in construction and identical physical characteristics of all new houses, a light gauge steel structure was chosen by the architects. The building site was selected by the houseowners in discussion with the architects to address the requirements of the houseowners and optimize shade throughout the day. The raised concrete foundation serves as the ground floor and can be easily cleaned. The ground floor includes a protected lockable storage area to reduce rodent infestation and to provide a feeling of security. Each of the 3 doors comes with a spring powered closing mechanism to minimize the time that the doors stay open. The frequency of opening and closing of the door will be recorded by data loggers via sensors. The ground floor cooking area is screened to improved airflow and reduce the entry of flies. The kitchen includes a chimney to direct the flow of smoke away from the building. Rainwater is harvested in roof gutters, which drain into 2000 litre PVC water tanks. The outdoor latrines are constructed within a $10 \mathrm{~m}$ radius of the building, using a light gauge steel system with a flyproof, ventilated septic tank. Each house comes with a solar power system which provides electricity for light and mobile phone charging. The new homes are designed to minimize energy consumption through reduced use of concrete and optimise airflow and ventilation. By our estimates a novel design house uses $37 \%$ less embodied carbon, $41 \%$ less embodied energy, and $70 \%$ less concrete than a more traditional African home design of similar size ${ }^{18}$.

The construction of the 110 novel-design houses started at the end of 2018 and was completed in June 2021 when the houses were handed over to the new house owners. Each novel design house was matched to four traditional homes in the same village resulting in the recruitment of 440 control homes. A maximum of three new houses were built in each village to minimize the risk of mosquito diversion, i.e. mosquitoes prevented from entering an intervention house being diverted to a control house and thus inflating malaria transmission in control houses. The residual mosquito diversion is unlikely to increase exposure in unprotected homes ${ }^{19}$.

\section{Participant eligibility}

The randomization procedure started by identifying suitable villages based on the eligibility criteria described below.

Village eligibility criteria

- Size: A village of about 500 people or more (about 100 households), to make sure that we would have at least five households for randomization.

- Accessibility: Readily accessible throughout the year to assist during construction phase and surveillance.

- No competing health interventions: We selected a study area without plans for new, experimental health interventions. The routine government health interventions including the distribution of ITNs continues.

- No power supply: Lack of electricity is representative of most Tanzanian villages. 
- No water supply: Few Tanzanian villages have access to reliable water supplies.

Following the selection of 68 eligible villages a census of 14,600 households in the 68 villages was conducted, during which time data on household members and household structures were collected ${ }^{17}$. Data were entered in tablets and subsequently uploaded, stored and processed using KoBo Toolbox. Eligible households were selected according to the criteria below.

Household eligibility criteria:

- There is sufficient land on which to construct another house.

- At least two children under 13 years old (no lower age limit).

- Willing to participate in disease surveillance for three years.

- Traditional house design constructed with mud walls, thatched roof and dirt floor.

- Householders had access to a pit latrine.

- No power supply from the grid.

- No piped water supplied by the municipality.

\section{Randomization}

All households meeting the eligibility criteria were invited to participate in a randomization process in the form of a village lottery. A total of 862 households were eligible to participate in a lottery to select: firstly the 110 new house owners and secondly the 440 household to serve as comparison houses. The lottery was conducted during a public meeting at a central meeting place to which all villagers were invited. All households meeting the inclusion criteria were listed and cards with the names of the household's heads were inserted in identical envelopes. The envelopes were placed in a transparent bucket and mixed. The lottery consisted of two rounds where the winning envelope was picked by a literate school child of any age. In the first stage two envelopes were picked out of the bucket and the winners were offered a new house. During the second round of the lottery, eight households were randomly selected to participate in the study as comparison households. Comparison households will receive modifications of their homes such as a corrugate iron roof and water harvesting equipment after completion of the three-year surveillance period. All study families, control and intervention are enrolled in a health insurance scheme which assures free healthcare for the study duration. Each household meeting the eligibility criteria had an equal and fair chance of being selected to be part of the study (figure 2).

\section{Community engagement}

A hierarchical approach to engage the study population was chosen at the earliest contact with the prospective study communities. The study team sought the advice of the district government and based on their recommendations, approached the village leadership to explain the purpose and methods of the proposed study. The village leaders organized village meetings preceding census activities in the villages. Following the completion of the census all villagers were invited to meetings to explain the purpose of the 
lottery described below. During the construction phase there was regular contact with the new house owners and their neighbours. The construction team explained the activities and, where possible, asked the new houseowners to participate in the construction.

Following the hand-over of the new houses, the research team realized that envy among community members who had not been allocated a novel design house was hindering study participation. In response a multipronged community engagement plan was implemented prior to the start of active surveillance activities. A professional drama group developed plays originally meant to be enacted as village theatre. Following COVID-19 related restrictions, the script was transformed into a radio show "Samoe" with 20 episodes of 20 minutes each, describing the adventures of a young couple after participating in the lottery and winning a new-design home. Due to the popularity of sports and particularly football, the research team formed a football club which participated in weekly matches in the study villages. Additional sports activities were added for all day village "bonanzas" on weekends. In conjunction with the sports days, village open-air dances were organized in-between and after sports activities. Rest periods between activities are used to introduce the research team and explain the purpose and the methods of the study.

\section{Clinical data collection}

Each household and household members will be assigned unique study Identification numbers. Clinical data will be collected at the household level from children under 13 years of age. The follow-up period is for three years from 2022 to 2024. Clinical data collection will comprise active and passive surveillance, annual malaria surveys and annual anthropometric surveys.

For the active case surveillance trained research assistants will visit each child weekly and collect disease and travel history, temperature, and a rapid diagnostic test to detect $P$. falciparum infections if there is history of fever since the last visit. A history of diarrhoea defined by 3 loose bowel movement or a bloody loose bowel movement within a 24-hour period or acute respiratory tract infection defined as an increased respiratory rate for age and/or difficulty breathing will be elicited at each household visit. To help the family remember what happened during the week, we have designed a disease symptoms card which the head of household or any other adult in the house completes if fever, diarrhoea, or acute respiratory tract infection is observed in a study child. The research assistants will refer ill children to a health care facility, as needed.

Passive case detection will be conducted in all health care facilities serving the study population, documented in an earlier healthcare uptake / treatment seeking behaviour study (manuscript in preparation). Study participants will be asked to identify themselves when entering the health care facility to benefit from the health insurance provided by the project. Staff working in the facilities will be trained on how to document the data of study participants. Research assistants will visit the health facility and electronically record the relevant information. 
To detect any difference in parasite prevalence between participants in the two study arms annual malaria surveys will be conducted. Dried blood spots (DBS) and a rapid test for malaria will be collected from all children participating in this study. This survey will be done four times throughout the 3-year study period (at the beginning, and once every year for three years). The DBS will be stored in freezers until processing for $\mathrm{PCR}^{20,21}$.

To detect any difference in growth between pre-school study participants in the two study arms anthropometric measurements (weight, height, and mid arm circumference) will be collected at the beginning of the study and then annually.

\section{Microbiology}

Starting in the second year of surveillance, nasal swabs will be collected from children with symptoms consistent with acute respiratory tract infections and rectal swabs will be collected from children with diarrhoea. In addition, a swab will be collected from a child without symptoms residing in a control house. The swabs will be transported in an appropriate transport medium at ambient temperature to the study hub in Mtwara town where the specimens will be stored at $-20 \mathrm{C}$ until final processing using multiplex $\mathrm{PCR}^{22}$. An air sampler will be used to collect standardized air samples to detect pathogens in air samples ${ }^{23}$.

\section{Entomology}

Standard CDC light traps will be used to estimate the potential exposure to malaria mosquitoes in the bedrooms of study children in 110 novel design houses and their nearest comparison house from October 2021 to December 2023. The study area will be divided into seven geographically separated clusters, six each with 16 Star homes and 16 control houses and one with 14 Star homes and 14 control houses. Each cluster will further be divided into four sub-clusters, each with eight houses: four Star homes and four nearest neighbors control houses. The light traps will be suspended $1 \mathrm{~m}$ from ground at the foot end of a bed where a study child. Collections will be made from one cluster each week, with a different sub-cluster sampled on four consecutive nights each week, Monday night to Thursday night. At the end of the week all houses will be sampled in each cluster (i.e. 32 houses). The sequence in which clusters will be visited was randomly determined. The clusters will be visited in the same sequence throughout the study period, so that each cluster will be visited in a seven-week cycle. Malaria vectors collected in the study houses will be counted and compared between the intervention and control houses and then packed in the Eppendorf tubes containing silica gel and submitted to the laboratory, for further analyses. The mosquitoes will be identified by microscopy and the numbers of An. gambiaes.l., An. funestus s.l., and other species will be recorded. The presence of sporozoites will be identified using an enzyme-linked immunosorbent assay ${ }^{24}$, and An. gambiae s.I. females, typed by $\mathrm{PCR}^{25}$.

The abundance and species of domestic flies will be assessed using baited traps installed in the cooking area of selected households, outdoors and in latrines to assess the difference of fly densities between the 
two study arms. The traps will be positioned on the kitchen floor, in the corner furthest from the main door. Research assistants will collect the captured flies each evening.

\section{Social science}

A mixed-method approach will be deployed for the assessment of acceptance and the use of each component of the novel design house (i.e., kitchen, storage area, bedroom, latrine, water collection facility). Data will be collected using focus group discussions (FGDs), in-depth interviews (IDIs), and a structured questionnaire-based survey at the beginning of the study and after one to two years. In-depth interviews will be conducted with approximately 18 selected heads of the households as well as selected members of the community (Community leaders, Health workers, religious leaders etc.) to get their perspectives of the new house designs (design elements, construction materials, aesthetic appearance, etc.) and use of the house. FGDs will be conducted with a group of eight to 10 participants including respondents from both study arms and other selected members of the community to explore the community perception of the novel design houses. Approximately 10 FGDs will be conducted with approximately a total of 100 participants taking part in the discussions. The construction costs of novel design houses will be estimated and used to assess incremental costs and cost-effectiveness of the interventions.

\section{Architecture and environment}

Data loggers will be used to record environmental data. The data will be recorded in intervals throughout the follow-up period and downloaded weekly or monthly intervals depending on the storage capacity of the device. Rainfall, temperature, relative humidity, and wind speed \& direction will be recorded using an Automatic Weather Station placed in the centre of the study area. Indoor temperature, relative humidity, and carbon dioxide concentrations will be recorded in the bedrooms of study homes in eight Star homes and eight neighbouring traditional study homes each week. PM2.5 will be monitored in the kitchens and bedrooms of study and control houses. Readings will be made every 30 minutes for three consecutive nights. GasLab data loggers will be positioned at the foot end of the study child's, 1 metre above the floor. Aerosol dispersion will be studied using Computational Fluid Dynamics to digitally model the aerosols movements in space. The model will be informed by the environmental data and further qualified by targeted on-site registrations.

\section{Mitigation of bias and confounding}

The intervention - a novel house design - makes it impossible to conduct a blinded trial. Researchers and village residents are aware who lives in an intervention and a control house. The awareness of the intervention allocation can lead to differences in the detection of diseases and hence introduce bias. The study team aware of this risk will take all possible steps to prevent differences in data capture between study arms. Observer bias will be minimised wherever feasible. 
Stratified randomisation by village will reduce the likelihood of imbalances due to differences in geographic characteristics between study groups. In addition, data on potential confounding factors will be collected and corrected for in the analysis. Laboratory technicians will be blinded to the identity and intervention status of the subjects. Collector bias will be reduced by using standard light traps and fly traps which do not rely on the ability of the fieldworker to collect specimens. Trap catches will be examined by a different person blinded to the trap location.

The study participants can only benefit from the house design intervention if they make use of the new houses and sleep upstairs. Children moving between houses and not regularly sleeping upstairs in study houses will be identified during weekly interviews with the household head or guardian and censored in the analysis.

\section{Study endpoints}

\section{Primary endpoints}

Incidence of malaria will be calculated in children under 13 years participating in the study for three years, using the number of detected cases as the numerator and the period contributed by each child residing in the study area as the denominator. These data will be analysed and used to determine the relative risk of malaria among children living in the novel design houses compared with those living in traditional subSaharan African homes.

\section{Secondary endpoints}

Incidence of respiratory tract infections and diarrhoea diseases will be calculated, and relative risks determined among children living in the novel design houses compared with those living in traditional African homes.

P. falciparum prevalence: The prevalence of malaria parasitaemia among children living in novel design houses and traditional homes will be compared in annual malaria surveys. Finger prick blood specimens will be collected from all children participating in the study for $P$. falciparum rapid diagnostic test and dried blood spots for qPCR.

Difference in growth parameters: Weight, height, body mass index (BMI) and mid-upper arm

circumference of all children participating in the study will be recorded at baseline, annually, and at end of the study. Mean changes in growth parameters will be compared among children living in intervention and control houses.

Difference in disease severity: Differences in disease severity between children residing in novel design houses and traditional homes will be analysed by comparing hospitalization rates.

Microbiology: Differences in the frequency of pathogens detected in symptomatic and healthy children will be used to calculate attributable fractions of pathogens and compared between intervention and 
control houses. Pathogens detected by air sampling will be compared between intervention and control houses.

Mean number of malaria vectors/light trap/night: The number and type of mosquitoes from novel design houses and traditional homes will be determined at various time points throughout the study period. This will be used to assess differences and similarities of mosquito densities between novel design houses and traditional homes as a proxy for measuring exposure to malaria transmission.

Environmental measurements: Indoor climate and other relevant environmental metrics will be measured for 3 years using data loggers. This will be used to compare indoor temperature, humidity, carbon dioxide, $\mathrm{PM}_{2.5}$ particle pollution and door opening and closing in both study arms.

Acceptability of interventions: The acceptance of novel design houses will be assessed using qualitative and quantitative research methods. Interviews will be conducted at 6 months after moving into the houses, and after 1 to 2 years.

Bednet use: Household heads of traditional and novel design houses will be interviewed annually to estimate the use of ITNs.

Durability: Yearly inspections of all study houses to assess durability and modifications of the various components of the building structure.

Economics: Comparison of construction costs and cost benefits for intervention and control houses.

Aerosol dispersion: Comparing the risk of becoming infected with SARS CoV-2 (COVID-19) via aerosols from another occupant of the same household, between the intervention houses and the control houses.

\section{Sample size calculations}

Our sample size estimates are based on malaria incidence observed in the RTS,S cohort phase 3 trial $^{26}$. Malaria epidemiology in Tanzania is quickly changing and the main burden of disease has moved from young children aged 5-17 months to older children and adolescents. Assuming malaria episodes of $10 / 100$ children/year in the control and a $30 \%$ reduction from 10 to 7 malaria episodes/100 children/year, a coefficient of variation (CV) of the outcome of 0.25 , as recommended by Hayes and Moulton $2009^{27}$, detecting the difference in incidence rates with $80 \%$ power and testing at $5 \%$ significance level, we would need 105 clusters (households) with three child/years of observations per household per arm. To allow for loss to follow-up we plan to recruit 110 households to the intervention arm. Since analysis requires adjustment for clustering by village and household ${ }^{28}$, power simulations were performed using a random effects model to assess what ratio of traditional to novel-design households provides adequate power. For every intervention house we will recruit four control houses, which will provide at least $80 \%$ power at the $5 \%$ level of significance to detect a $33 \%$ effect size. Since ICCs that accommodate village and household level clustering are rarely reported, we compensated clustering from both levels by choosing a 
conservatively high ICC of 0.6 . Because malaria is rarer than either respiratory diseases or diarrhoeal diseases in the study population, the sample size should suffice to measure a $33 \%$ reduction in all diseases under investigation at a $5 \%$ level of significance and $80 \%$ power. The sample size was performed in Stata 15 using the following basic command: clustersampsi, samplesize rates r1(.1) r2(.07) $\mathrm{m}(10)$ cluster $\mathrm{cv}(0.25)$ alpha(0.05) beta(0.8) base correl $(0.6)$. The simulation results are shown in figure below. Simulations were performed in Stata and each scenario simulated 5,000 times and the empirical power was obtained. We considered 20, 40, 80 and 110 households in the intervention.

\section{Entomological outcomes}

We postulate that the intervention homes will reduce indoor collections of malaria vectors by at least $50 \%$. Based on a recent study using light traps in the Kilombero Valley, Tanzania the mean number of female An. gambiae s.I. per trap in a house was $10.4(S D=21.5)$. Computer simulations based on a negative binomial distribution suggest with $89 \%$ power at the $5 \%$ level of significance to detect a $50 \%$ reduction in indoor-entering mosquitoes (i.e. An. gambiae s.I.) 110 Star Homes and 440 control houses will be needed. We propose to collect a sample from each house every seven weeks each year for three years.

\section{Acceptability outcomes}

The total number of interviews will depend on when data saturation is reached, i.e., when it is unlikely to lead to the identification of any significant new issue or novel insight. We hope to reach saturation with 18 in-depth interviews with heads of households and 10 focus group discussions with approximately 8 to 10 participants per group.

\section{Analytical plan}

Incidence of malaria, respiratory tract infections and diarrhea diseases: Protective efficacy against clinical malaria, pneumonia and diarrhea diseases will be assessed by comparing incidence rates between the two study arms adopting an intention-to-treat analysis. Children within each household will be monitored using active and passive case detection hroughout the study period. After treatment for malaria, the child will not be considered at risk for the following four weeks and this period will be censored. History of travel and nights spent away from the designated household will be captured during weekly surveillance visits and time at risk will be censored for such periods. Initial unadjusted analyses will be based on the comparison of the incidence rates between the two arms. Formal analyses will use a mixed effects Poisson model to test the difference in incidence rate between the two arms allowing for the repeated measurements within a house, a village and the effect of year using of confidence intervals that account for the highest level of clustering ${ }^{28}$. With multiple control and intervention houses within each village the "intervention*village" interaction will also be quantified. Possible confounders such as age of child, gender, ethnicity and rainfall will be tested using the mixed effects model. A mixed effects Negative Binomial model will be considered in case of overdispersion in the mixed effects Poisson model. A comparison of time to infection (both first and repeat) will be examined using a survival 
analysis approach. Initial analyses will be based on a Kaplan-Meier curves with further adjustment for confounders performed using a Cox regression model.

Entomology (Malaria transmission): Differences in malaria transmission experienced in the two groups will be made by comparing the mean number of malaria mosquitoes (as a proxy for transmission) caught indoors in houses between the study arms. Generalised estimating equations will be used to estimate differences in numbers of indoor-resting mosquitoes, adjusting for repeated measures within houses and possible covariates. We will compare the entomological inoculation rate in both arms. Human behavioural studies will also be carried out to compare when people move indoors and outdoors of the house, since the new housing intervention may change the way individuals use a house, which changes their exposure risk. Observers will record human movement including the age and gender of individuals with their identity anonymised.

Clinical (P. falciparum, respiratory infections and diarrhoeal diseases): $P$. falciparum prevalence in children will be estimated at the start of the transmission season through cross-sectional survey. Falciparum malaria, respiratory and enteric disease incidence will be estimated based on active and passive surveillance and compared between study arms. The incidence of all-cause febrile illness during the three-year study and age-adjusted growth rates will be estimated in children under five years old.

Acceptability: Perceptions of the novel design house owners and the general community regarding the aesthetic appearance and convenience of the houses, use of different spaces in the house, its design elements and construction materials will be summarized to provide an overall impression of acceptance of the novel design houses. Proportion of owners of control houses who choose to have home improvements at the end of the study will be determined.

ITN use: The proportion of children sleeping under ITNs between study arms at different times of the year will be compared.

Architectural: Indoor temperatures, humidity, $\mathrm{CO}_{2}$ and $\mathrm{PM}_{2.5}$ particles pollutions will be quantified using data loggers and compared between the two study arms. This will provide data regarding level of air pollution between novel design and traditional homes and whether it correlates with respiratory tract infections. The durability of the novel design houses will be assessed by yearly inspections of houses. In traditional houses maintenance records will be kept to allow comparisons between the study arms.

Aerosol dispersion will be modelled using Computational Fluid Dynamics (CFD). The CFD simulations will provide insights into the effect of various environmental parameters on the transport/dispersion of aerosols between indoors and outdoors in novel design houses and traditional houses respectively, and subsequently allow us to assess the relative risk of airborne transmission of COVID-19 between inside and outside of a home.

\section{Discussion}


The idea that better house design leads to better health is for most readers intuitively convincing. Evidence in support of this notion is however scant and generally considered of questionable quality because it is not derived from randomised controlled trials ${ }^{29}$. The rapid growth of a population in Africa provides an opportunity for research on specific house design elements that are associated with health benefits. From 2000-2015, the prevalence of homes in sub-Saharan Africa with improved water and sanitation, and of durable construction doubled from $11-23 \%$ of houses ${ }^{30}$.The results from this study could potentially influence the design of future housing in sub-Saharan Africa.

A systematic review of 90 studies published in 2015 found overwhelming evidence that house improvements, described as "modern houses", have a protective effect against malaria ${ }^{31}$. Four randomised controlled trials of house screening have been published, with three trials demonstrating protection against malaria ${ }^{32-34}$ and one outlier ${ }^{35}$.

Whether housing improvements can protect against respiratory tract infections and diarrhoea is less obvious. A systematic review published in 2021 "indicated a potential benefit of home environmental interventions in preventing overall respiratory tract infections" ${ }^{36}$. In contrast a "big data" approach reanalysing diverse surveys that included more than 800,000 children found that housing improvements reduced the odds of childhood malaria and diarrhoea but failed to detect any protection against acute respiratory tract infections ${ }^{37}$. Considering the disparity of interventions and geography, this systematic review may not be ideal to predict whether novel house designs can protect against respiratory tract infections in rural Tanzania. Similarly reanalysing a range of surveys conducted for purposes other than the assessment health benefits of housing improvements may neither be specific enough to accurately distinguish relevant housing improvements nor reliable enough to detect acute respiratory tract infections.

The strategies for prevention of diarrhoea includes the provision of safe drinking water, sanitation, and hygiene (WASH), all of which can be addressed by home improvements. Yet the evidence of whether WASH strategies can indeed prevent diarrhoea and thereby improve childhood growth is at best equivocal. Three large, recent trials ${ }^{38-40}$ of unprecedented scale and cost found mixed effects on diarrhoea and no protection against stunting ${ }^{41}$. We hypothesise that controlling multiple childhood diseases will have multiplicative health benefits. If correct, we anticipate less severe disease and less cumulative uncomplicated disease will ultimately result in less malnutrition and better growth parameters of children living in novel houses compared with those living in traditional houses.

The majority of homes currently constructed in sub-Saharan Africa have concrete floors, concrete walls and a corrugate iron roof. A house design pattern emerging throughout sub-Saharan Africa is reminiscent of housing developments in the US or Europe but is heavily dependent on air-conditioning to maintain a comfortable indoor climate in a hot-tropical climate. Cement production is highly energyintensive as is air conditioning ${ }^{42}$. The design of the houses in the study is a notable exception from this trend since the use of concrete and the need for air-conditioning are intentionally minimised. 
Although unique and without precedent our study has several limitations. Firstly, it is impossible to blind the study subjects and field researchers from the intervention. We will try to address this handicap by conscientiously assuring that the same procedures are applied to all participants and that those analysing samples in the laboratory are blinded to the source of the sample. Secondly, since our primary outcome is disease incidence the capture of all disease episodes is of critical importance. This will be achieved through a combination of active cases detection supported by passive case detection in all health facilities in the study catchment area. It is possible to miss disease episodes if the guardians of study participants fail to inform the research assistants and seek either no healthcare at all or seek healthcare outside the recognized system. Building rapport and ultimately gaining the trust of the families living in study houses will be the key strategy for the capture of all disease episodes. Thirdly, some families are sceptical about using the novel houses. Community sensitization and engagement will continue throughout the trial period to ensure full compliance. A multipronged community engagement programme is underway addressing the fears of using the houses to facilitate the understanding between the study communities and the researchers. Finally, and perhaps most importantly the interventions can only work if the study participants sleep and more broadly live in their assigned homes. If children assigned to novel design homes sleep in the homes of neighbours or relatives, they cannot be protected by the novel home design. Data will hence be collected on household use to assess how often children sleep in the novel design houses. This type of dilution of protection may be most intense for respiratory tract infections and diarrhoea where study participants can be infected at any time of the day including while attending schools without a safe water supply, and where sanitation and ventilation have not been improved. As falciparum malaria is predominantly transmitted by endophagic and endophilic vectors in sub-Saharan Africa most of the transmission occurs at night at home. Hence the impact on malaria transmission of novel home designs may be least diluted. Potential protection against respiratory tract infections and diarrhoea could be reduced through infections acquired outside the home.

\section{Protocol Version}

$1^{\text {st }}$ June 2020 , version 2.0

\section{Trial status}

The random allocation of houses was completed in 2018. The house construction was completed by June 2021. The houses were handed over and the new residents moved in between June 2021 and January 2021. The disease surveillance started in January 2022. The surveillance will be completed in December 2024. The analysis and publication of the most pertinent findings should be completed by December 2025.

\section{Conclusions}

This protocol describes an open-label, household randomized controlled trial to assess the protective efficacy of a novel design house against three major childhood diseases, malaria, respiratory tract 
infections and diarrhoea diseases compared to those living in traditional homes. This trial provides an opportunity to collect high grade evidence for the benefits of a range of housing interventions. The trial comes at a unique moment of what could be the onset of an intense population growth phase on the African continent. People need homes and rapid population growth will result in massive formal and informal housing construction. Making relatively small modifications in the design of houses to reduce the risk of mosquito entry, improve air quality, and assure safe water supply and sanitation could result in long term health benefits affecting present and future generations.

\section{Abbreviations}

ITNs, insecticide-treated nets; $\mathrm{Cl}$, confidence interval; PM2.5, atmospheric particulate matter (PM) with a diameter of less than 2.5 micrometers; PAPfPR, population adjusted Plasmodium falciparum parasite rate; Fig, figure; DBS, dried blood spot; PCR, polymerase chain reaction; $C D C$, centres for disease control; An, anopheles; FGD, focus group discussion; IDI, in depth interview; CO2, carbon dioxide; CFD, computational fluid dynamics;

\section{Declarations}

\section{Ethics approval and consent to participate}

The investigators will ensure that this study is conducted in compliance with the current revision of the Declaration of Helsinki. All researchers working in this project must be trained in Good Clinical Practice. The study protocol has been approved by the National Research Ethics Committee of Tanzania on $17^{\text {th }}$ June 2021 (reference NIMR/HQ/R.8a/Vol.IX/3695) and Oxford Tropical Research Ethics Committee $2^{\text {nd }}$ July 2020 (reference \#533-20). Approval for any important protocol modifications will be sought from both ethics committees.

Written informed consent was obtained from the head of household during the eligibility assessment and re-affirmed prior to the start of disease surveillance of the children. In addition, assent is obtained from children 10 years of age and older. Participants or parents/guardian have the right to withdraw their child/children from the study at any time. Children reaching 13 years of age will be censored in the analysis. This is a dynamic cohort such that children leaving the study site will be withdrawn, whereas newborn or older children under 13 years joining the household will be invited to participate in the study.

\section{Consent for publication}

All authors read and approved the final manuscript. For the purpose of Open Access, the authors have applied a CC BY public copyright licence to any Author Accepted Manuscript version arising from this submission.

\section{Availability of data and materials}


The study data will be available upon request to the Mahidol Oxford Tropical Medicine Research Unit Data Access Committee (http://www.tropmedres.ac/data-sharing) for researchers and following the Mahidol Oxford Tropical Medicine Research Unit data access policy (http://www.tropmedres.ac/_asset/file/datasharing-policy-v1-1.pdf). Queries and applications for datasets should be directed to Rita Chanviriyavuth (rita@tropmedes.ac).

\section{Competing interests}

The authors declare that they have no competing interests.

\section{Sponsor}

University of Oxford, University of Oxford Richard Doll Building, Old Road Campus, Oxford OX3 7LF, United Kingdom

\section{Funding}

The study is funded by the Hanako Foundation, Singapore. Hanako plays no role in study design; collection, management, analysis, and interpretation of data; writing of the report; and the decision to submit the report for publication.

\section{Authors' contributions}

SM, JK, SWL, LVS conceived and designed the study. SM, JD, LvS designed the clinical surveillance. AM, FO, SWL designed the entomological assessment. JM, CP designed the qualitative assessment. TC, JK designed the assessment of the architectural component of the study, JD designed the anthropometric surveillance. MM wrote the clinical analytic plan and JB the entomological analytical plan. ND and AA supervise the study implementation. All authors contributed the writing of the manuscript.

\section{Acknowledgements}

First and foremost, we thank the children and their families who have agreed to participate in the study. We thank the Mtwara regional and District administration for agreeing to have us and for their continuous support of the project.

\section{References}

1. Pison G. The Population of the World. Population \& Societies 2017; (547).

2. UN. The Sustainable Development Goals Report. https://unstatsunorg/sdgs/report/2021/TheSustainable-Development-Goals-Report-2021pdf 2021.

3. Knudsen J, Seidlein Lv. Healthy homes in tropical zones, Improving rural housing in Asia and Africa. Stuttgart London: Stuttgart : Axel Menges; 2014. 
4. von Seidlein L, Ikonomidis K, Bruun R, et al. Airflow attenuation and bed net utilization: observations from Africa and Asia. Malaria journal 2012; 11: 200.

5. Pulford J, Hetzel MW, Bryant M, Siba PM, Mueller I. Reported reasons for not using a mosquito net when one is available: a review of the published literature. Malaria journal 2011; 10: 83.

6. Charlwood JD, Pinto J, Ferrara PR, et al. Raised houses reduce mosquito bites. Malaria journal 2003; 2(1): 45.

7. Carrasco-Tenezaca M, Jawara M, Abdi MY, et al. The relationship between house height and mosquito house entry: an experimental study in rural Gambia. J R Soc Interface 2021; 18(178): 20210256.

8. Jatta E, Carrasco-Tenezaca M, Jawara M, et al. Impact of increased ventilation on indoor temperature and malaria mosquito density: an experimental study in The Gambia. J R Soc Interface 2021; 18(178): 20201030.

9. Piddock KC, Gordon SB, Ngwira A, et al. A cross-sectional study of household biomass fuel use among a periurban population in Malawi. Ann Am Thorac Soc 2014; 11(6): 915-24.

10. Dherani M, Pope D, Mascarenhas M, Smith KR, Weber M, Bruce N. Indoor air pollution from unprocessed solid fuel use and pneumonia risk in children aged under five years: a systematic review and meta-analysis. Bull World Health Organ 2008; 86(5): 390-8C.

11. Darvesh N, Das JK, Vaivada T, et al. Water, sanitation and hygiene interventions for acute childhood diarrhea: a systematic review to provide estimates for the Lives Saved Tool. BMC Public Health 2017; 17(Suppl 4): 776.

12. Hartinger SM, Lanata $\mathrm{CF}$, Hattendorf J, et al. Improving household air, drinking water and hygiene in rural Peru: a community-randomized-controlled trial of an integrated environmental home-based intervention package to improve child health. Int J Epidemiol 2016; 45(6): 2089-99.

13. Das JK, Bhutta ZA. Global challenges in acute diarrhea. Curr Opin Gastroenterol 2016; 32(1): 18-23.

14. von Seidlein L, Ikonomidis K, Mshamu S, et al. Affordable house designs to improve health in rural Africa: a field study from northeastern Tanzania. Lancet Planet Health 2017; 1(5): e188-e99.

15. Alegana V, Macharia P, Muchiri S, et al. Plasmodium falciparum parasite prevalence in East Africa: Updating data for malaria stratification. PLOS GPH 2021: https://doi.org/10.1371/journal.pgph.0000014.

16. This_is_Mtwara.| Mtwara Region [Internet]. [cited 2021 Oct 19]. Available from: . http://wwwmtwaragotz/mtwara.

17. Mshamu S, Peerawaranun $P$, Kahabuka $C$, et al. Old age is associated with decreased wealth in rural villages in Mtwara, Tanzania: findings from a cross-sectional survey. Trop Med Int Health 2020; 25(12): 1441-9.

18. Ingvartsen. http://ingvartsen.dk/star-homes/tjv53tlf1hup4xhx5081cgknbb89so.

19. Clarke SE, Bogh C, Brown RC, Pinder M, Walraven GE, Lindsay SW. Do untreated bednets protect against malaria? Trans $R$ Soc Trop Med Hyg 2001; 95(5): 457-62. 
20. Singh B, Cox-Singh J, Miller AO, Abdullah MS, Snounou G, Rahman HA. Detection of malaria in Malaysia by nested polymerase chain reaction amplification of dried blood spots on filter papers. Trans R Soc Trop Med Hyg 1996; 90(5): 519-21.

21. Win AA, Imwong M, Kyaw MP, et al. K13 mutations and pfmdr1 copy number variation in Plasmodium falciparum malaria in Myanmar. Malaria journal 2016; 15: 110.

22. Duong VT, Tu LTP, Tuyen HT, et al. Novel multiplex real-time PCR assays reveal a high prevalence of diarrhoeagenic Escherichia coli pathotypes in healthy and diarrhoeal children in the south of Vietnam. BMC Microbiol 2020; 20(1): 192.

23. Horve PF, Dietz L, Northcutt D, Stenson J, Van Den Wymelenberg K. Evaluation of a bioaerosol sampler for indoor environmental surveillance of Severe Acute Respiratory Syndrome Coronavirus 2. PLoS One 2021; 16(11): e0257689.

24. Wirtz RA, Duncan JF, Njelesani EK, et al. ELISA method for detecting Plasmodium falciparum circumsporozoite antibody. Bull World Health Organ 1989; 67(5): 535-42.

25. Scott JA, Brogdon WG, Collins FH. Identification of single specimens of the Anopheles gambiae complex by the polymerase chain reaction. Am J Trop Med Hyg 1993; 49(4): 520-9.

26. Rts SCTP. Efficacy and safety of RTS,S/AS01 malaria vaccine with or without a booster dose in infants and children in Africa: final results of a phase 3 , individually randomised, controlled trial. Lancet 2015; 386(9988): 31-45.

27. Hayes RJ, Moulton LH. Cluster Randomised Trials. Oxfordshire: Routledge; 2017.

28. Bottomley C, Kirby MJ, Lindsay SW, Alexander N. Can the buck always be passed to the highest level of clustering? BMC Med Res Methodol 2016; 16: 29.

29. WHO. https://www.who.int/publications/i/item/9789241550376.

30. Tusting LS, Bisanzio D, Alabaster G, et al. Mapping changes in housing in sub-Saharan Africa from 2000 to 2015. Nature 2019; 568(7752): 391-4.

31. Tusting LS, Ippolito MM, Willey BA, et al. The evidence for improving housing to reduce malaria: a systematic review and meta-analysis. Malaria journal 2015; 14: 209.

32. Kirby MJ, Ameh D, Bottomley C, et al. Effect of two different house screening interventions on exposure to malaria vectors and on anaemia in children in The Gambia: a randomised controlled trial. Lancet 2009; 374(9694): 998-1009.

33. Getawen SK, Ashine T, Massebo F, Woldeyes D, Lindtjorn B. Exploring the impact of house screening intervention on entomological indices and incidence of malaria in Arba Minch town, southwest Ethiopia: A randomized control trial. Acta Trop 2018; 181: 84-94.

34. Ng'ang'a PN, Okoyo C, Mbogo C, Mutero CM. Evaluating effectiveness of screening house eaves as a potential intervention for reducing indoor vector densities and malaria prevalence in Nyabondo, western Kenya. Malaria journal 2020; 19(1): 341.

35. Pinder M, Bradley J, Jawara M, et al. Improved housing versus usual practice for additional protection against clinical malaria in The Gambia (RooPfs): a household-randomised controlled trial. 
Lancet Planet Health 2021; 5(4): e220-e9.

36. Kua KP, Lee D. Home environmental interventions for prevention of respiratory tract infections: a systematic review and meta-analysis. Rev Environ Health 2021; 36(3): 297-307.

37. Tusting LS, Bottomley C, Gibson H, et al. Housing Improvements and Malaria Risk in Sub-Saharan Africa: A Multi-Country Analysis of Survey Data. PLoS Med 2017; 14(2): e1002234.

38. Luby SP, Rahman M, Arnold BF, et al. Effects of water quality, sanitation, handwashing, and nutritional interventions on diarrhoea and child growth in rural Bangladesh: a cluster randomised controlled trial. Lancet Glob Health 2018; 6(3): e302-e15.

39. Null C, Stewart CP, Pickering AJ, et al. Effects of water quality, sanitation, handwashing, and nutritional interventions on diarrhoea and child growth in rural Kenya: a cluster-randomised controlled trial. Lancet Glob Health 2018; 6(3): e316-e29.

40. Humphrey JH, Mbuya MNN, Ntozini R, et al. Independent and combined effects of improved water, sanitation, and hygiene, and improved complementary feeding, on child stunting and anaemia in rural Zimbabwe: a cluster-randomised trial. Lancet Glob Health 2019; 7(1): e132-e47.

41. Cumming O, Arnold BF, Ban R, et al. The implications of three major new trials for the effect of water, sanitation and hygiene on childhood diarrhea and stunting: a consensus statement. BMC Med 2019; 17(1): 173.

42. Calder B. Architecture: From Prehistory to Climate Emergency. London: Pelican; 2021.

\section{Figures}

\section{Figure 1}

Diagram illustrating critical elements of the novel rural African house design.

To reduce malaria, enteric and respiratory tract infections and to improve indoor climate and safety our latest design (Fig. 1) includes the following critical structural components:

1) building orientated to provide optimal shading throughout the day to keep the house cooler at night. $(\rightarrow$ indoor climate)

2) lightweight and durable roof with partially closed eaves to reduce the entry of malaria vectors. ( $\rightarrow$ malaria, indoor climate)

3) facade and openings screened to reduce insect entry while assuring airflow. ( $\rightarrow$ malaria, diarrhoeal diseases, indoor climate)

4) raised concrete ground floor which can be easily cleaned increases hygiene and reduces the risk of enteric and soil-transmitted infections. ( $\rightarrow$ diarrhoeal diseases) 
5) a screened indoor cooking area with means to remove smoke to reduce indoor pollution. ( $\rightarrow$ respiratory infections)

6) a protected lockable storage area to reduce rodent infestations and provide a feeling of security. ( $\rightarrow$ safety)

7) sleeping areas with bednets raised to the first floor improving airflow, comfort while reducing mosquito density. ( $\rightarrow$ malaria, indoor climate)

8) a water harvesting system which allows the collection of rainwater from the roof, filtering, and covered storage. ( $\rightarrow$ diarrhoeal diseases)

9) an outdoor fly-proof latrine. ( $\rightarrow$ diarrhoeal diseases)

10) solar power providing electric light at night. ( $\rightarrow$ safety).

Figure 2

assembly of study households and participants

Figure 3

Simulated power calculations showing power by ratios of intervention to control houses, assuming a protective efficacy of $33 \%$.

\section{Supplementary Files}

This is a list of supplementary files associated with this preprint. Click to download.

- SPIRITcheckliststarhomes.docx 